\title{
Early biliary drainage is associated with favourable outcomes in critically-ill patients with acute cholangitis
}

\author{
Mohammed Aboelsoud ${ }^{1}$, Osama Siddique ${ }^{1}$, Alexander Morales ${ }^{1}$, Young Seol ${ }^{1}$, Mazen Al-Qadi ${ }^{1,2}$ \\ ${ }^{1}$ Department of Medicine, Memorial Hospital of Rhode Island, Alpert Medical School of Brown University, Providence, USA \\ ${ }^{2}$ Division of Pulmonary, Critical Care and Sleep Medicine, Providence, USA
}

Gastroenterology Rev 2018; 13 (1): 16-21

DOI: https://doi.org/10.5114/pg.2018.74557

Key words: acute cholangitis, biliary drainage, endoscopic retrograde cholangiopancreatography, Tokyo Guidelines 2013, critically-ill.

Address for correspondence: Osama Siddique MD, Department of Medicine, Memorial Hospital of Rhode Island, Alpert Medical School

of Brown University, 111 Brewster St, 02860 Pawtucket, USA, phone: +1 401729 2258, e-mail: osama_siddique@brown.edu

\begin{abstract}
Introduction: Acute cholangitis (AC) is a clinical condition that requires prompt medical management with IV fluids, antibiotics, and biliary drainage (BD). The optimal timing for BD remains unclear.

Aim: To investigate the effect of biliary drainage timing on clinical outcomes in AC.

Material and methods: We conducted a retrospective study of patients with AC admitted to the ICU using the Multiparameter Intelligent Monitoring in Intensive Care III (MIMIC-III) database. Emergency department to BD time, hospital death, length of stay (LOS), and severity scores were extracted from the database. We investigated the effect of BD timing on mortality rates, persistent organ failure, and LOS.

Results: A total of 177 patients were included; $50 \%$ were males; median age was 75 years, in-hospital mortality was $9.6 \%$, mean time-to-ERCP was $32 \mathrm{~h}$ (range: 0.42-229.6) with 76\% meeting the Tokyo Guidelines (TG13) criteria for severe cholangitis, and median Simplified Acute Physiology Score II (SAPS II) was 42 (IQR: 33-51). Using $24 \mathrm{~h}$ as a cut-off, patients who underwent $\mathrm{BD} \leq 24 \mathrm{~h}$ had less persistent organ failure $(\mathrm{OR}=0.49 ; 95 \% \mathrm{Cl}: 0.26-0.96, p=0.040)$, shorter ICU LOS (3.25 vs. 4.95 days, $p=0.040)$, shorter hospital LOS (7.71 vs. 13.57 days, $p=0.001)$, but no difference in either in-hospital mortality $(\mathrm{OR}=0.47$, $95 \% \mathrm{Cl}: 0.17-1.29, p=0.146)$ or 28 -day mortality $(\mathrm{OR}=0.61,95 \% \mathrm{Cl}: 0.24-1.53, p=0.297)$.

Conclusions: In critically-ill patients with acute cholangitis, early biliary drainage $\leq 24 \mathrm{~h}$ is associated with less persistent organ failure and shorter length of stay but had no effect on patient survival.
\end{abstract}

\section{Introduction}

Acute cholangitis $(A C)$ is a clinical condition caused by infection of an obstructed biliary system. Despite the development of increasingly sophisticated treatment modalities, mortality remains near $11 \%$ [1-4]. Treatment of $A C$ requires timely intravenous fluid resuscitation, antibiotic administration, and biliary drainage (BD). However, up to $80 \%$ of patients respond to medical management alone [5-8]. Endoscopic retrograde cholangiopancreatography (ERCP) has historically been the procedure of choice for $\mathrm{BD}$ in cholangitis, although percutaneous biliary drainage (PBD) or surgical drainage may still be used in selected patients [9]. Endoscopic retrograde cholangiopancreatography has been proven to be safer than surgical drainage. Nevertheless, ERCP is a complex procedure that is resource-intensive and has its own inherent risks of technical failure, and procedural and anaesthesia-related adverse events. Urgent, or even emergent $\mathrm{BD}$, is believed to source-control cholangitis through promotion of immunoglobulin $A$, antibiotic excretion into bile, and also by reducing cholangio-venous reflux $[10,11]$. The current guidelines of Tokyo 2013 recommend urgent/emergent biliary drainage for moderate and severe cholangitis, without a specified time frame in which this should be accomplished [8]. Most experts recommend biliary decompression within 48-72 h. A few studies have been conducted that aim to delineate the effect of timing at which endoscopic $\mathrm{BD}$ is undertaken, and consequent patient outcomes [3, 12-14]. Determining the optimal timing for BD as well as other predictors of outcome would certainly aid clinicians in providing optimal care, and subsequently help to improve patient outcomes. 


\section{Aim}

Herein, we conducted a retrospective study to investigate the effect of BD timing on mortality rates, organ failure, and length of stay as a primary aim. The secondary aim was to identify predictors of in-hospital mortality.

\section{Material and methods \\ Study design}

We conducted a retrospective study to investigate the effect of biliary drainage timing on clinical outcomes in critically-ill patients with acute cholangitis.

\section{Study population}

We used the Multiparameter Intelligent Monitoring in Intensive Care (MIMIC-III) research database developed by researchers from the Laboratory for Computational Physiology at Massachusetts Institute of Technology (MIT), Cambridge, MA, USA, and the Department of Medicine at the Beth Israel Deaconess Medical Centre (BIDMC) Boston, MA, USA. The database has detailed information about intensive care unit (ICU) patient stays, including high-resolution vital sign trends and waveforms, laboratory data, therapeutic interventions, discharge summaries, radiology reports, and International Classification of Diseases, $9^{\text {th }}$ Revision (ICD-9) codes for all patients admitted to BIDMC ICU between 2001 and 2012. Patients were de-identified in a Health Insurance Portability and Accountability Act-compliant manner. The institutional review boards of BIDMC and MIT approved the use of the MIMIC-III database [15].

We included all adult patients ( $>18$ years) between 2001 and 2012 admitted directly to the intensive care unit from the emergency department with AC. Patients who were transferred from other facilities or did not have AC on admission were excluded. We searched the database for patients who were admitted with ICD-9 codes of 576.1 and 576.2, and had either ERCP or PBD. Subsequently, patients were reviewed to verify compatibility with the Tokyo 2013 diagnostic criteria for AC. Patients who did not meet the diagnostic criteria or had missing data were excluded.

\section{Study variables}

Demographic data including age, sex, and race were obtained for each patient. Clinical data included vital signs, fluid intake/output, time and type of biliary drainage (ERCP vs. PBD), registration time at the emergency department, and hospital/ICU admission and discharge times. Biliary drainage timing was calculated as the difference between ED registration time and time of the procedure. Laboratory data included alanine aminotransferase, aspartate aminotransferase, alkaline phosphatase, albumin, total bilirubin, international normalised ratio (INR), lactate, platelets count, blood urea nitrogen (BUN), creatinine, and presence of bacteraemia. For grading of severity, we used SAPS-II score and TG13 severity criteria.

\section{Study outcomes}

The primary study outcomes were in-hospital and 28-day mortality. The secondary outcomes were ICU length of stay (LOS), hospital LOS, and persistent organ failure. Persistent organ failure was defined as having any of the following for $>48 \mathrm{~h}$ : hypotension requiring vasopressors of any dose, acute kidney injury (defines as $\geq 1$.5-fold rise in creatinine from baseline, need for renal replacement therapy), $\mathrm{PaO}_{2} / \mathrm{FiO}_{2}<300$, or need for mechanical ventilation.

\section{Statistical analysis}

Age, vital signs, biochemical profile, SAPS-II, and LOS were defined as continuous variables, while ethnicity, gender, TG13 severity, and in-hospital and 28-day mortality were defined as categorical variables. Continuous variables were reported as mean \pm standard deviation or median with interquartile range when appropriate; categorical variables are reported as percentages. For biliary drainage timing, we examined the effect of BD timing (as a continuous variable) on persistent organ failure, in-hospital, and 28-day mortality using logistic regression. Then we examined the effect of $\mathrm{BD}$ timing (as a categorical variable) using two cut-off points: before or after 24 and 48 h. Comparisons between groups for categorical variables were evaluated using Pearson's $\chi^{2}$ test for contingency, and for continuous variables, a two-sided $t$ test was used.

We conducted a supplementary analysis to identify predictors for the primary study outcome. A multivariate analysis was conducted using a logistic regression model. The model included potential variables from univariate analysis with $p<0.1$, as well as those with clinical significance even if not reaching the pre-defined statistical significance. In the event of co-linearity between variables, only one variable was included. All statistical tests and/or confidence intervals $(\mathrm{Cl})$, as appropriate, were performed at $\alpha=0.05$. All reported $p$-values were two sided and rounded to three decimal places. Statistical analysis was performed using JMP Pro by SAS Institute.

\section{Results}

\section{Patient characteristics}

We identified 1155 patients with ICD-9 codes of 576.1 and 576.2 between 2001 and 2013. Out of those 


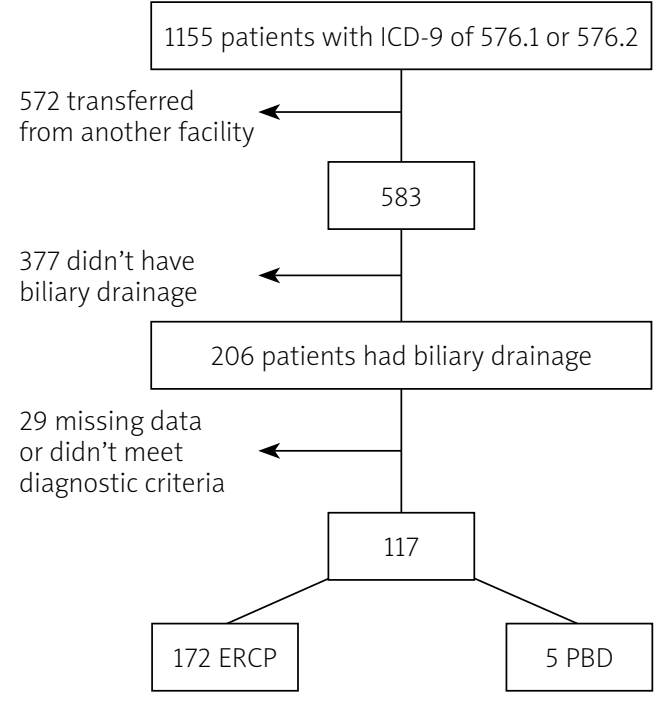

Figure 1. Flow diagram of study population $E R C P$ - endoscopic retrograde cholangio-pancreatography, $P B D$ percutaneous biliary drainage.

Table I. Baseline characteristics

\begin{tabular}{lc} 
Variable & Value \\
\hline Age, median (IQR) [years] & $75(62-84)$ \\
\hline Men, $n$ (\%) & $89(50)$ \\
\hline TG13 severity, $n$ (\%): & $135(76)$ \\
\hline Severe & $19(11)$ \\
\hline Moderate & $23(13)$ \\
\hline Mild & $42(33-51)$ \\
\hline SAPS-II, median (IQR) & $52(29)$ \\
\hline Bacteraemia, $n$ (\%) & $172(97)$ \\
\hline ERCP, $n$ (\%) & $5(3)$ \\
\hline PBD, $n$ (\%) & $32(0.42-229.6)$ \\
\hline Timing to biliary drainage, mean (range) [h] & $122(69)$ \\
\hline Biliary drainage within, $n$ (\%): & $26(15)$ \\
\hline $24 \mathrm{~h}$ & $29(16)$ \\
\hline $24-48 \mathrm{~h}$ & \\
\hline$\geq 48 \mathrm{~h}$ &
\end{tabular}

IQR - interquartile range, TG13 - Tokyo Guidelines 2013, SAPS-II - Simplified Acute Physiology Score-II, ERCP - endoscopic retrograde cholangio-pancreatography, $P B D$ - percutaneous biliary drainage.

patients, only 177 satisfied our inclusion criteria. We excluded 572 patients who were transferred from other facilities, as well as 377 patients who did not undergo biliary drainage. An additional 29 patients were excluded for having missing data or not fulfilling the diagnostic criteria (Figure 1).
The baseline characteristics for the whole cohort are summarised in Table I. The median age of the group was 75 years (IQR: 62-84 years), and 76\% had severe cholangitis per TG13 criteria with median SAPS-II score of 42 (IQR: $33-5$ ). Bacteraemia was evident in $29 \%$ of patients. The majority of patients (97\%) underwent ERCP as the chosen modality for biliary drainage, while $3 \%$ underwent PBD. The mean time to biliary drainage was 32 h (range: $0.42-229.6$ h), with $69 \%$ of patients undergoing biliary drainage within the first $24 \mathrm{~h}$. Persistent organ failure (> 48 h) was observed in $32.77 \%$ of patients. The overall in-hospital and 28-day mortality rates were $9.6 \%$ and $12.4 \%$, respectively. Median (IQR) for ICU and hospital LOS were 1.94 (1.39-3.62) and 6.76 (4.03-11.34) days, respectively. Patients' characteristics based on biliary drainage timing using cut-offs of 24 and $48 \mathrm{~h}$ are summarised in Table II. Overall, the subgroups were comparable except for age, bacteraemia, where the early ERCP ( $\leq 24 \mathrm{~h}$ and $\leq 48 \mathrm{~h})$ subgroups were older and had more bacteraemia compared to the late ERCP ( $>24 \mathrm{~h}$ and $>48 \mathrm{~h}$ ) groups, respectively. Also, the early ERCP subgroup had lower platelets compared to the late ERCP group.

\section{Clinical outcomes by biliary drainage timing}

The clinical outcomes based on timing of BD are summarised in Table III and Figure 2. When using $24 \mathrm{~h}$ as a cut-off, we found that those who underwent early $\mathrm{BD}(\leq 24 \mathrm{~h})$ had less persistent organ failure $(\mathrm{OR}=0.49$, 95\% Cl: 0.26-0.96, $p=0.040$ ), shorter ICU LOS (3.25 vs. 4.95 days, $p=0.040)$, and shorter hospital LOS (7.71 vs. 13.57 days, $p=0.001)$, but no difference in either in-hospital mortality $(\mathrm{OR}=0.47,95 \% \mathrm{Cl}: 0.17-1.29$, $p=0.146)$ or 28 -day mortality $(\mathrm{OR}=0.61,95 \% \mathrm{Cl}$ : $0.24-1.53, p=0.297)$.

When using $48 \mathrm{~h}$ as a cut-off, those who underwent early BD (< $48 \mathrm{~h}$ ) had shorter hospital LOS (8.61 vs. 14.24 days, $p=0.002)$, but no difference in persistent organ failure $(O R=0.76,95 \% \mathrm{Cl}: 0.33-1.74, p=0.521)$, ICU LOS (3.49 vs. 5.26 days, $p=0.190$ ) in-hospital mortality $(\mathrm{OR}=0.6,95 \% \mathrm{Cl}: 0.18-2.0, p=0.4230)$, or 28 -day mortality $(\mathrm{OR}=0.62,95 \% \mathrm{Cl}: 0.21-1.85, p=0.409)$. Using BD timing as a continuous variable, earlier BD was associated with shorter hospital LOS ( $p=0.003)$, but there was no significant effect on other clinical outcomes.

\section{Predictors of in-hospital mortality}

On univariate analysis, we found associations between in-hospital morality and serum albumin $(p=0.002)$, total bilirubin $(p=0.008)$, lactate levels $(p=0.011)$, and SAPS-II $(p=0.003)$ on admission. Subsequently, we conducted a multivariate model that 
Table II. Patient characteristics by timing of biliary drainage

\begin{tabular}{|c|c|c|c|c|}
\hline Parameter & $\leq 24 \mathrm{~h}(n=122)$ & $>24 \mathrm{~h}(n=55)$ & $\leq 48 \mathrm{~h}(n=148)$ & $>48 \mathrm{~h}(n=29)$ \\
\hline Age, mean (SD) [years] & $74.51(14.22)^{\star}$ & $68.87(14.84)^{\star}$ & $73.88(14.14)^{\star}$ & $67.03(15.84)^{\star}$ \\
\hline Men, $n(\%)$ & $56(45.90)$ & $33(60.00)$ & $70(47.30)$ & $19(65.52)$ \\
\hline SAPS-II, median (IQR) & $43.99(15.29)$ & $42.04(14.13)$ & $43.30(15.31)$ & $43.72(13.08)$ \\
\hline Severe, $n(\%)$ & $92(75.41)$ & $43(78.18)$ & $111(75)$ & $24(82.76)$ \\
\hline Bacteraemia, $n$ (\%) & $27(22.13)^{*}$ & $25(45.45)^{\star}$ & $37(25)^{*}$ & $15(51.72)^{\star}$ \\
\hline \multicolumn{5}{|c|}{ Admission laboratory tests, mean (SD): } \\
\hline AST & $298.12(483.21)$ & $211.21(263.92)$ & $282.25(447.55)$ & $211.58(306.95)$ \\
\hline ALT & $225.88(180.40)$ & $173.96(184.61)$ & $219.43(181.32)$ & $157.27(185.17)$ \\
\hline ALP & $38.42(362.35)$ & $298.81(237.53)$ & $366.33(349.21)$ & 307.54 (196.34) \\
\hline Albumin & $2.89(0.63)$ & $2.88(0.63)$ & $2.89(0.62)$ & $2.91(0.67)$ \\
\hline Total bilirubin & $5.40(3.85)$ & $5.09(4.95)$ & $5.16(3.70)$ & $6.15(6.39)$ \\
\hline INR & $1.63(0.97)$ & $1.76(1.03)$ & $1.62(0.94)$ & 1.89 (1.19) \\
\hline Leukocyte count & $16.46(8.22)$ & $18.15(11.43)$ & $16.54(8.18)$ & $19.26(13.75)$ \\
\hline BUN & $27.23(16.52)$ & $33.89(30.80)$ & $27.44(18.26)$ & $38.79(34.83)$ \\
\hline Creatinine & $1.48(1.21)$ & $1.81(2.05)$ & $1.49(1.19)$ & $2.06(2.61)$ \\
\hline Lactate & $2.96(2.63)$ & $2.83(2.30)$ & $2.87(2.46)$ & $3.18(2.92)$ \\
\hline Platelet count & $184.55(94.29)^{*}$ & $226.47(138.86)^{\star}$ & 188.95 (103.42) & 242.10 (139.89) \\
\hline
\end{tabular}

${ }^{*} P<0.05$. SAPS-II - Simplified Acute Physiology Score-II, AST - aspartate aminotransferase, ALT - alanine aminotransferase, ALP - alkaline phosphatase, INR - international normalised ratio, BUN - blood urea nitrogen.

Table III. Clinical outcomes by biliary drainage timing

\begin{tabular}{lcccccc} 
Parameter & $\langle\mathbf{2 4} \mathrm{h}(\boldsymbol{n}=\mathbf{1 2 2})$ & $\boldsymbol{>} \mathbf{2 4} \mathrm{h}(\boldsymbol{n}=\mathbf{5 5})$ & $\boldsymbol{P}$-value & $<\mathbf{4 8} \mathrm{h}(\boldsymbol{n}=\mathbf{1 4 8})$ & $\boldsymbol{>} \mathbf{4 8} \mathrm{h}(\boldsymbol{n}=\mathbf{2 9})$ & $\boldsymbol{P}$-value \\
\hline Persistent organ failure $\geq 48 \mathrm{~h}(\%)$ & 27.87 & 43.64 & 0.040 & 31.76 & 37.93 & 0.521 \\
\hline ICU LOS, mean (SD) [days] & $3.25(4.15)$ & $4.95(6.50)$ & 0.040 & $3.49(4.59)$ & $5.26(6.81)$ & 0.190 \\
\hline Hospital LOS, mean (SD) [days] & $7.71(6.77)$ & $13.57(11.90)$ & 0.001 & $8.61(7.47)$ & $14.24(14.04)$ & 0.002 \\
\hline In-hospital mortality (\%) & 7.38 & 14.55 & 0.146 & 8.78 & 13.79 & 0.423 \\
\hline 28-day mortality (\%) & 10.66 & 16.36 & 0.297 & 11.49 & 17.24 & 0.409
\end{tabular}

LOS - length of stay, SD - standard deviation.

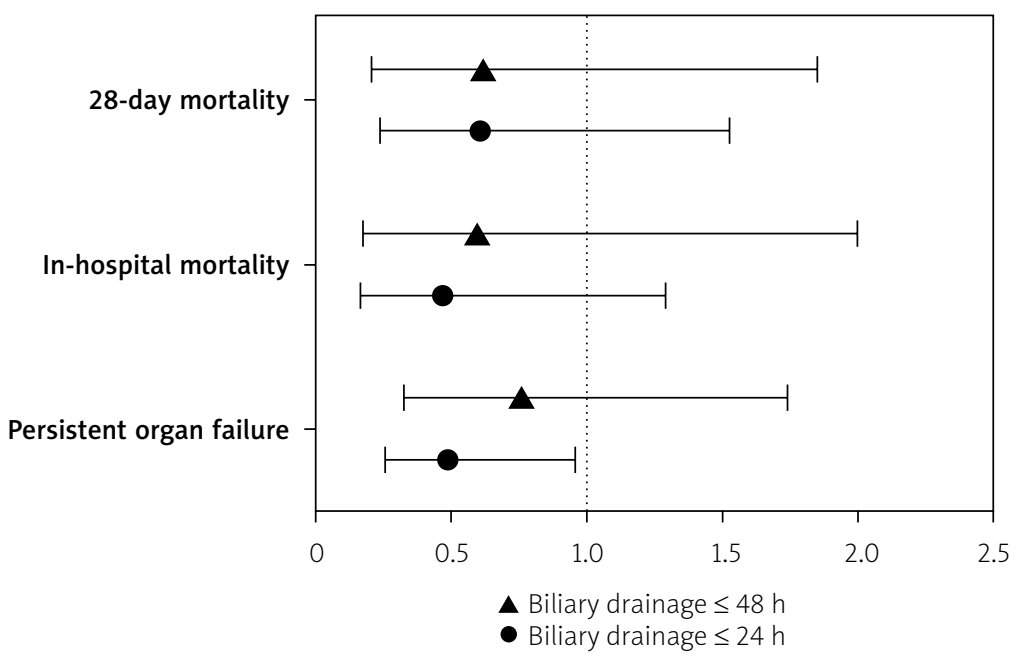

Figure 2. Odds ratios for clinical outcomes based on timing of biliary drainage 
included the previous variables in addition to age, BD timing (at the two cut-offs 24 and $48 \mathrm{~h}$ ), and bacteraemia for clinical significance and to adjust for differences in subgroups. Albumin was found to be the most prominent predictor for in-hospital mortality, with an adjusted OR of 3.54 (95\% Cl: 1.12-14.07) for each $1 \mathrm{mg} / \mathrm{dl}$ drop in serum albumin $(p=0.03)$.

\section{Discussion}

In our study, we investigated the effect of BD timing on clinical outcomes using two cut-off points: 24 and $48 \mathrm{~h}$. Overall, there was less persistent organ failure, shorter LOS, and less mortality with early drainage (using either 24 or 48 h) although the effect on mortality did not reach statistical significance. The benefit was more prominent with earlier BD $(<24 \mathrm{~h})$ for persistent organ failure, and both ICU and hospital LOS. The decision to pursue this study came from the paucity of literature evaluating the effect of BD timing in AC. Surprisingly, various $\mathrm{Gl}$ societies recommend specific timing for biliary drainage based solely on expert opinion, with a relative lack of clinical evidence backing such guidelines. This is probably a result of the relative rarity of the disease. Many patients with AC respond to medical management alone, subsequently precluding an emergent need for biliary drainage. The BD procedures are often performed on an elective basis. Since the majority of our patients (84\%) had biliary drainage within the first $48 \mathrm{~h}$, we felt that using 24 and $48 \mathrm{~h}$ as cut-off points would be appropriate. Overall, our results are in concordance with expert opinion in that BD drainage probably improves clinical outcomes. Thus far, few studies have been conducted to investigate the optimal timing for BD. Chak et al. showed that early ERCP $(<24 \mathrm{~h})$ is associated with shorter length of hospital stay. This study, however, did not evaluate other clinical outcomes such as organ failure and mortality [16]. In two different studies, delayed ERCP ( $>72 \mathrm{~h}$ ) was associated with longer LOS and composite outcome (including persistent organ failure, ICU admission, and mortality) rather than evaluating each of these outcomes separately $[3,4]$. Lee et al. also showed that delayed ERCP (> $48 \mathrm{~h}$ ) was associated with persistent organ failure and longer LOS. There was also increased mortality with delayed ERCP, but this did not reach statistical significance [13]. As demonstrated in our study, earlier biliary drainage is associated with improved outcomes in general, but the optimal timing is still unknown. Our study included $76 \%$ with sever cholangitis, which could explain why early biliary drainage $<24 \mathrm{~h}$ rather than $<48 \mathrm{~h}$ was associated with better outcomes. Biliary decompression was proven to decrease cholangio-venous reflux and subsequently decrease bile and serum endotoxin levels [11]. It also promotes the biliary excretion of IgA and antibiotics [10]. Theoretically, achieving these effects with early BD would improve organ failure and shorten hospital stay times. Surprisingly, we did not find the expected effect of early BD on mortality. However, most of the previously conducted studies did not demonstrate a clear effect of early biliary drainage on mortality as a single outcome. We hope to see more studies evaluating the effect of early BD on mortality specifically. The severity of illness and hypoalbuminaemia were independent predictors of mortality in $A C$, but timing of BD was not.

Our study had several limitations. The retrospective nature of our study makes it susceptible to bias. We adopted very rigorous inclusion and exclusion criteria, which impacted the sample size, but we still hold strong in our belief that this was necessary to obtain reliable results. Our study also included only critically-ill patients from a single centre, which should caution us from generalising the results to a larger population. However, this study has much strength. It is one of the few studies that systemically assessed clinical outcomes of patients with acute cholangitis. Also, our study included a preponderance of patients with severe cholangitis, thus requiring biliary drainage, as opposed to those patients presenting with mild cholangitis.

\section{Conclusions}

Early BD $(<24 \mathrm{~h})$ is associated with less persistent organ failure, shorter ICU, and hospital length of stay in critically ill patients with acute cholangitis. Higher severity scores and lower albumin levels are independently associated with increased mortality.

\section{Conflict of interest}

The authors declare no conflict of interest.

\section{References}

1. Rosing DK, De Virgilio C, Nguyen AT, et al. Cholangitis: analysis of admission prognostic indicators and outcomes. Am Surg 2007; 73: 949-54.

2. Salek J, Livote E, Sideridis K, et al. Analysis of risk factors predictive of early mortality and urgent ERCP in acute cholangitis. J Clin Gastroenterol 2009; 43: 171-5.

3. Khashab MA, Tariq A, Tariq U, et al. Delayed and unsuccessful endoscopic retrograde cholangiopancreatography are associated with worse outcomes in patients with acute cholangitis. Clin Gastroenterol Hepatol 2012; 10: 1157-61.

4. Navaneethan U, Gutierrez NG, Jegadeesan R, et al. Factors predicting adverse short-term outcomes in patients with acute cholangitis undergoing ERCP: a single center experience. World J Gastrointest Endosc 2014; 6: 74-81. 
5. Hui CK, Lai KC, Yuen MF, et al. Acute cholangitis: predictive factors for emergency ERCP. Aliment Pharmacol Ther 2001; 15: $1633-7$.

6. Kinney TP. Management of ascending cholangitis. Gastrointest Endosc Clin N Am 2007; 17: 289-306.

7. Kimura Y, Takada T, Kawarada Y, et al. Definitions, pathophysiology, and epidemiology of acute cholangitis and cholecystitis: Tokyo Guidelines. J Hepatobiliary Pancreat Surg 2007; 14: 15-26.

8. Kiriyama S, Takada T, Strasberg SM, et al. TG13 guidelines for diagnosis and severity grading of acute cholangitis (with videos). J Hepatobiliary Pancreat Sci 2013; 20: 24-34.

9. Lai EC, Mok FP, Tan ES, et al. Endoscopic biliary drainage for severe acute cholangitis. N Engl J Med 1992; 326: 1582-6.

10. Sung JJ, Leung JC, Tsui CP, et al. Biliary IgA secretion in obstructive jaundice: the effects of endoscopic drainage. Gastrointest Endosc 1995; 42: 439-44.

11. Lau JY, Chung SC, Leung JW, et al. Endoscopic drainage aborts endotoxaemia in acute cholangitis. Br J Surg 1996; 83: 181-4.

12. Mok SR, Mannino CL, Malin J, et al. Does the urgency of endoscopic retrograde cholangiopancreatography (ERCP)/percutaneous biliary drainage (PBD) impact mortality and disease related complications in ascending cholangitis? (DEIM-I study). J Interv Gastroenterol 2012; 2: 161-7.

13. Lee F, Ohanian E, Rheem J, et al. Delayed endoscopic retrograde cholangiopancreatography is associated with persistent organ failure in hospitalised patients with acute cholangitis. Aliment Pharmacol Ther 2015; 42: 212-20.

14. Inamdar S, Sejpal DV, Ullah M, et al. Weekend vs. weekday admissions for cholangitis requiring an ERCP: comparison of outcomes in a national cohort. Am J Gastroenterol 2016; 111: 405-10.

15. Saeed M, Villarroel M, Reisner AT, et al. Multiparameter Intelligent Monitoring in Intensive Care II (MIMIC-II): a public-access intensive care unit database. Crit Care Med 2011; 39: 952-60.

16. Chak A, Cooper GS, Lloyd LE, et al. Effectiveness of ERCP in cholangitis: a community-based study. Gastrointest Endosc 2000; 52: 484-9.

Received: 13.07 .2016

Accepted: 28.03 .2017 